\title{
Existence Theorems for Elliptic Quasi-Variational Inequalities in Banach Spaces
}

\author{
Risei Kano*, Nobuyuki Kenmochi ${ }^{\dagger}$ and Yusuke Murase ${ }^{\#}$ \\ *,\# Department of Mathematics, Graduate School of Science \& Technology \\ Chiba University, 1-33 Yayoi-cho, Inage-ku, Chiba, 263-8522 Japan \\ ${ }^{\dagger}$ Department of Mathematics, Faculty of Education, Chiba University \\ 1-33 Yayoi-cho, Inage-ku, Chiba, 263-8522 Japan
}

\begin{abstract}
A class of quasi-variational inequalities (QVI) of the elliptic type is studied in Banach spaces. The concept of QVI was ealier introduced by A. Bensoussan and J. L. Lions [2] and its general theory was evolved by many mathematicians, for instance, see $[7,9,13]$ and a monograph [1]. In this paper we give a generalization of the existence theorem due to J. L. Joly and U. Mosco $[6,7]$ from not only the view-point of the nonlinear operator theory, but also the application to nonlinear variational inequalities including partial differential operators. In fact, employing the compactness argument based on the Mosco convergence (cf.[11]) for convex sets and the graph convergence for nonlinear operators, we shall prove an abstract existence result for our class of QVI's. Moreover we shall give some new applications to QVI's arising in the material science.
\end{abstract}

\section{Introduction}

Let $X$ be a real reflexive Banach space and $X^{*}$ be its dual. We assume that $X$ and $X^{*}$ are strictly convex and denote by $\langle\cdot, \cdot\rangle$ the duality pairing between $X^{*}$ and $X$. Given a nonlinear operator $A$ from $X$ into $X^{*}$, an element $g^{*} \in X^{*}$ and a closed convex subset $K$ of $X$, the variational inequality is formulated as a problem to find $u$ in $X$ such that

$$
u \in K, \quad<A u-g^{*}, u-w>\leq 0, \quad \forall w \in K .
$$

Variational inequality has been studied by many mathematicians, for instance see J. L. Lions and G. Stampacchia [10], F. Browder [5], H. Brézis [4] and their references.

The concept of quasi-variational inequality was introduced by A. Bensoussan and J. L. Lions [2] in order to solve some problems in the control theory. Given an operator 
$A: X \rightarrow X^{*}$, an element $g^{*} \in X^{*}$ and a family $\{K(v) ; v \in X\}$ of closed convex subsets of $X$, the quasi-variational inequality is a problem to find $u$ in $X$ such that

$$
u \in K(u), \quad<A u-g^{*}, u-w>\leq 0, \quad \forall w \in K(u) .
$$

As is seen from (1.2), the constraint $K(u)$ for the quasi-variational inequality depends upon the unknown $u$, which causes one of main difficulties in the mathematical treatment of quasi-variational inequalities. The theory of quasi-variational inequality has been evolved for various classes of the mapping $v \rightarrow K(v)$ and the linear or nonlinear operator $A: X \rightarrow X^{*}$; see for instance $[6,7,13]$, in which two approaches to quasi-variational inequalities were proposed. One of them is the so-called monotonicity method in Banach lattices $X$ (cf. [13]), and for the mapping $v \rightarrow K(v)$ the monotonicity condition

$$
\min \left\{w_{1}, w_{2}\right\} \in K\left(v_{1}\right), \quad \max \left\{w_{1}, w_{2}\right\} \in K\left(v_{2}\right), \quad \text { if } v_{1}, v_{2} \in X \text { with } v_{1} \leq v_{2},
$$

is required, and an existence result for (1.2) is proved with the help of a fixed point theorem in Banach lattices. Another is the so-called compactness method in which some compactness properties are required for the mapping $v \rightarrow K(v)$ such as $K\left(v_{n}\right)$ converges to $K(v)$ in the Mosco sense, if $v_{n} \rightarrow v$ weakly in $X$ as $n \rightarrow \infty$. In this framework, an existence result for (1.2) was shown by J. L. Joly and U. Mosco [7]. However this result seems not enough from some points of applications. The objective of this paper is to generalize the result in [7] to the case that $A: X \rightarrow X^{*}$ is the multivalued pseudo-monotone operator, $A u=\tilde{A}(u, u)$, generated by a semimonotone operator $\tilde{A}: X \times X \rightarrow X^{*}$. In such a case our quasi-variational inequality is of the form: Find $u \in X$ and $u^{*} \in X^{*}$ such that

$$
u \in K(u), u^{*} \in A u, \quad<u^{*}-g^{*}, u-w>\leq 0, \quad \forall w \in K(u) .
$$

This generalization (1.4) is new and enables us to apply it to the following quasi-variational inequality arising in the elastic-plastic torsion problem for visco-elastic material : Find $u \in H_{0}^{1}(\Omega)$ and $\tilde{u} \in L^{2}(\Omega)$ satisfying

$$
\left\{\begin{array}{c}
|\nabla u| \leq k_{c}(u) \text { a.e. on } \Omega, \tilde{u} \in \beta(u) \text { a.e. on } \Omega, \\
\sum_{i, j=1}^{N} \int_{\Omega} a_{i j}(x, u) \frac{\partial u}{\partial x_{i}} \frac{\partial(u-w)}{\partial x_{j}} d x+\int_{\Omega} \tilde{u}(u-w) d x \leq \int_{\Omega} f(u-w) d x, \\
\forall w \in H_{0}^{1}(\Omega) \text { with }|\nabla w| \leq k_{c}(u) \text { a.e. on } \Omega,
\end{array}\right.
$$

where $\Omega$ is a bounded smooth domain in $\mathbf{R}^{N}, f$ is given in $L^{2}(\Omega), k_{c}(\cdot)$ is a positive, smooth and bounded function on $\mathbf{R}$ and $\beta(\cdot)$ is a maximal monotone graph in $\mathbf{R} \times \mathbf{R}$ with linear growth at $\pm \infty$. In this case our abstract result is applied to

$$
K(v):=\left\{w \in H_{0}^{1}(\Omega) ;|\nabla w| \leq k_{c}(u) \text { a.e. on } \Omega\right\}
$$

and

$$
\tilde{A}(v, u):=-\sum_{i, j=1}^{N} \frac{\partial}{\partial x_{j}}\left(a_{i j}(x, v) \frac{\partial u}{\partial x_{i}}\right)+\beta(u) ;
$$

it should be noted that the family $\left\{K(v) ; v \in H_{0}^{1}(\Omega)\right\}$ given by (1.6) does not satisfy the monotonicity condition (1.3), and that the term $\beta(u)$ of $(1.7)$ is in general multivalued. 
Therefore, (1.5) is a new application in the respect that the differential form in (1.7) is quasi-linear and the additional term is multivalued in general.

\section{Main results}

Let $\mathrm{X}$ be a real Banach space and $X^{*}$ be its dual space, and assume that $\mathrm{X}$ and $X^{*}$ are strictly convex. We denote by $\langle\cdot, \cdot\rangle$ the duality pairing between $X^{*}$ and $X$, and by $|\cdot|_{X}$ and $|\cdot|_{X^{*}}$ the norms of $X$ and $X^{*}$, respectively. For various general concepts on nonlinear multivalued operators from $X$ into $X^{*}$, for instance, monotonicity and maximal monotonicity of operators, we refer to the monograph [1]. In this paper, we mean that operators are multivalued, in general. Given a general nonlinear operator $A$ from $X$ into $X^{*}$, we use the notations $D(A), R(A)$ and $G(A)$ to denote its domain, range and graph of $A$. In this paper, we formulate quasi-variational inequalities for a class of nonlinear operators, which is called semimonotone, from $X \times X$ into $X^{*}$.

Definition 2.1. An operator $\tilde{A}(\cdot, \cdot): X \times X \rightarrow X^{*}$ is called semimonotone, if $D(\tilde{A})=$ $X \times X$ and the following conditions (SM1) and (SM2) are satisfied:

(SM1) For any fixed $v \in X$ the mapping $u \rightarrow \tilde{A}(v, u)$ is maximal monotone form $D(\tilde{A}(v, \cdot))=X$ into $X^{*}$.

(SM2) Let $u$ be any element of $X$ and $\left\{v_{n}\right\}$ be any sequence in $X$ such that $v_{n} \rightarrow v$ weakly in $X$. Then, for every $u^{*} \in \tilde{A}(v, u)$ there exists a sequence $\left\{u_{n}^{*}\right\}$ in $X$ such that $u_{n}^{*} \in \tilde{A}\left(v_{n}, u\right)$ and $u_{n}^{*} \rightarrow u^{*}$ in $X^{*}$ as $n \rightarrow+\infty$.

Let $\tilde{A}: D(\tilde{A}):=X \times X \rightarrow X^{*}$ be a semimonotone operator. Then we define $A: D(A)=X \rightarrow X^{*}$ by putting $A u:=\tilde{A}(u, u)$ for all $u \in X$, which is called the operator generated by $\tilde{A}$.

Now, for an operator $A$ generated by semimonotone operator, any $g^{*} \in X^{*}$ and a mapping $v \rightarrow K(v)$ we consider a quasi-variational inequality, denoted by $P\left(g^{*}\right)$, to find $u \in X$ and $u^{*} \in X^{*}$ such that

$$
P\left(g^{*}\right) \quad u \in K(u), \quad u^{*} \in A u, \quad<u^{*}-g^{*}, w-u>\leq 0, \quad \forall w \in K(u) .
$$

Our main results of this paper are stated as follows.

Theorem 2.1. Let $\tilde{A}: D(\tilde{A})=X \times X \rightarrow X^{*}$ be a bounded semimonotone operator and $A$ be the operator generated by $\tilde{A}$. Let $K_{0}$ be a bounded, closed and convex set in $X$. Suppose that to each $v \in K_{0}$ a non-empty, bounded, closed and convex subset $K(v)$ of $K_{0}$ is assigned, and the mapping $v \rightarrow K(v)$ satisfies the following continuity properties (K1) and (K2) :

(K1) If $v_{n} \in K_{0}, v_{n} \rightarrow v$ weakly in $X$ (as $\left.n \rightarrow \infty\right)$, then for each $w \in K(v)$ there is a sequence $w_{n}$ in $X$ such that $w_{n} \in K\left(v_{n}\right)$ and $w_{n} \rightarrow w$ (strongly) in $X$.

(K2) If $v_{n} \rightarrow v$ weakly in $X, w_{n} \in K\left(v_{n}\right)$ and $w_{n} \rightarrow w$ weakly in $X$, then $w \in K(v)$. 
Then, for any $g^{*} \in X^{*}$, the quasi-variational inequality $P\left(g^{*}\right)$ has at least one solution $u$.

The following theorem is a slightly general version of Theorem 2.1.

Theorem 2.2. Let $\tilde{A}: D(\tilde{A})=X \times X \rightarrow X^{*}$ be a bounded semimonotone operator and $A$ be the operator generated by $\tilde{A}$. Suppose that to each $v \in X$ a non-empty, bounded, closed and convex subset $K(v)$ of $X$ is assigned and there is a bounded, closed and convex subset $G_{0}$ of $X$ such that

$$
K(v) \cap G_{0} \neq \emptyset, \quad \forall v \in X
$$

and

$$
\inf _{w^{*} \in A w} \frac{<w^{*}, w-v>}{|w|_{X}} \rightarrow \infty \text { as }|w|_{X} \rightarrow \infty \text { uniformly in } v \in G_{0} .
$$

Furthermore, the mapping $v \rightarrow K(v)$ satisfies the following condition (K'1) and the same condition (K2) as in Theorem 2.1.:

(K'1) If $v_{n} \rightarrow v$ weakly in $X$, then for each $w \in K(v)$ there is a sequence $w_{n}$ in $X$ such that $w_{n} \in K\left(v_{n}\right)$ and $w_{n} \rightarrow w$ in $X$.

Then, for any $g^{*} \in X^{*}$, the quasi-variational inequality $P\left(g^{*}\right)$ has at least one solution $u$.

In our proof of Theorems 2.1 and 2.2 we use some results on nonlinear operators of monotone type, which are mentioned below.

Proposition 2.1. Let $\tilde{A}: D(\tilde{A})=X \times X \rightarrow X^{*}$ be a semimonotone operator and let $A: X \rightarrow X^{*}$. Then, the following two properties $(a)$ and $(b)$ are satisfied:

(a) For any $v, u \in X, A(v, u)$ is a non-empty, closed, bounded and convex subset of $X^{*}$.

(b) Let $\left\{u_{n}\right\}$ and $\left\{v_{n}\right\}$ be sequences in $X$ such that $u_{n} \rightarrow u$ weakly in $X$ and $v_{n} \rightarrow v$ weakly in $X$ (as $n \rightarrow \infty)$. If $u_{n}^{*} \in \tilde{A}\left(v_{n}, u_{n}\right), u_{n}^{*} \rightarrow g$ weakly in $X^{*}$ and $\lim \sup _{n \rightarrow \infty}<$ $u_{n}^{*}, u_{n}>\leq<g, u>$, then $g \in \widetilde{A}(v, u)$ and $\lim _{n \rightarrow \infty}<u_{n}^{*}, u_{n}>=<g, u>$.

Proof. Property (a) immediately follows from the maximal monotonicity of $\tilde{A}(v, \cdot)$ for each $v \in X$. Now we show (b). Assume that $\left\{u_{n}\right\},\left\{v_{n}\right\}$ and $\left\{u_{n}^{*}\right\}$ are such as in the statement of (b), namely,

$$
u_{n} \rightarrow u \text { weakly in } X, \quad v_{n} \rightarrow v \text { weakly in } X, \quad u_{n}^{*} \rightarrow g \text { weakly in } X^{*}
$$

and

$$
u_{n}^{*} \in \tilde{A}\left(v_{n}, u_{n}\right), \quad \limsup _{n \rightarrow \infty}<u_{n}^{*}, u_{n}>\leq<g, u>.
$$

Now we note from (SM1) that

$$
<u_{n}^{*}-w_{n}^{*}, u_{n}-w>\geq 0, \quad \forall w \in X, \quad \forall w_{n}^{*} \in \tilde{A}\left(v_{n}, w\right) .
$$

For any $w \in X$ and any $w^{*} \in \tilde{A}(v, w)$, use (SM2) to choose a sequence $\left\{\tilde{w}_{n}^{*}\right\}$ with $\tilde{w}_{n}^{*} \in \tilde{A}\left(v_{n}, w\right)$ and $\tilde{w}_{n}^{*} \rightarrow w^{*}$ in $X^{*}$. Then, by substituting this sequence $w_{n}^{*}$ and letting $n \rightarrow \infty$ in (2.6) we have with the help of (2.4) and (2.5)

$$
<g-w^{*}, u-w>\geq 0, \quad \forall w \in X, \quad \forall w^{*} \in \tilde{A}(v, w) .
$$


This implies that $g \in \tilde{A}(v, u)$, since $\tilde{A}(v, \cdot)$ is maximal monotone. Corresponding to this $g \in \tilde{A}(v, u)$, by (SM2) choose a sequence $g_{n} \in \tilde{A}\left(v_{n}, u\right)$ such that $g_{n} \rightarrow g$ in $X^{*}$. Then, by taking $w=u$ and $w_{n}^{*}=g_{n}$ and letting $n \rightarrow \infty$ in (2.6) we obtain

$$
\liminf _{n \rightarrow \infty}<u_{n}^{*}, u_{n}-u>\geq \liminf _{n \rightarrow \infty}<g_{n}, u_{n}-u>=0 .
$$

namely, $\liminf \operatorname{in}_{n \rightarrow \infty}<u_{n}^{*}, u_{n}>\geq<g, u>$. Therefore, on account of (2.5), it holds that

$$
\lim _{n \rightarrow \infty}<u_{n}^{*}, u_{n}>=<g, u>.
$$

Thus (b) has been seen. $\diamond$

We consider a class of nonlinear operators $A: D(A)=X \rightarrow X^{*}$ satisfying the following properties (PM1) and (PM2):

(PM1) For any $u \in X, A u$ is a non-empty, closed, bounded and convex subset of $X^{*}$.

(PM2) Let $\left\{u_{n}\right\}$ be a sequence in $X$ such that $u_{n} \rightarrow u$ weakly in $X$. If $u_{n}^{*} \in A u_{n}$, $u_{n}^{*} \rightarrow g$ weakly in $X^{*}$ and $\limsup _{n \rightarrow \infty}<u_{n}^{*}, u_{n}>\leq<g, u>$, then $g \in A u$ and $\lim _{n \rightarrow \infty}<u_{n}^{*}, u_{n}>=<g, u>$.

This class of nonlinear operaors $A$ is called pseudo-monotone. The above proposition says that the operator generated by semimonotone $\tilde{A}$ is pseudo-monotone. As to pseudomonotone operators we refer to $[4,8]$ for fundamental results on their ranges.

Proposition 2.2. Let $A_{1}: D\left(A_{1}\right) \subset X \rightarrow X^{*}$ be a maximal monotone operator and $A_{2}: D\left(A_{2}\right)=X \rightarrow X^{*}$ be a maximal monotone operator. Suppose that

$$
\inf _{v_{1}^{*} \in A_{1} v, v_{2}^{*} \in A_{2} v} \frac{<v_{1}^{*}+v_{2}^{*}, v-v_{0}>}{|v|_{X}} \rightarrow \infty \quad \text { as }|v|_{X} \rightarrow \infty, v_{1} \in D\left(A_{1}\right) .
$$

Then $R\left(A_{1}+A_{2}\right)=X^{*}$.

For a proof of Proposition 2.2, see [4,5,8].

\section{Proof of main theorems}

We begin with the proof of Theorem 2.1.

Proof of Theorem 2.1: The theorem is proved in the following two steps: (A) The case when $\tilde{A}(v, \cdot)$ is strictly monotone from $X$ into $X^{*}$ for every $v \in X$; (B) The general case as in the statement of Theorem 2.1.

\section{(In the case of $(\mathrm{A})$ )}

Let $v$ be any element in $K_{0}$ and fix it. We consider the variational inequality with state constraint $K(v)$, namely, to find $u \in X$ and $u^{*} \in X^{*}$ such that

$$
u \in K(v), \quad u^{*}(v) \in \tilde{A}(v, u), \quad<u^{*}(v)-g^{*}, u-v>\leq 0, \quad \forall w \in K(v) .
$$


This problem is written in the following form equivalent to (3.1):

$$
g^{*} \in \tilde{A}(v, u)+\partial I_{K(v)}(u)
$$

where $\partial I_{K(v)}(\cdot): D\left(\partial I_{K(v)}\right) \rightarrow X^{*}$ is the subdifferential of the indicator function of $K(v)$, i.e.

$$
I_{K(v)}(z):= \begin{cases}0 & \text { if } z \in K(v) \\ \infty & \text { if } z \in X-K(v)\end{cases}
$$

note that $\partial I_{K(v)}$ is maximal monotone. It follows from Proposition 2.2 that $R(\tilde{A}(v, \cdot)+$ $\left.\partial I_{K(v)}\right)=X^{*}$; in fact, the coerciveness condition of Proposition 2.2 for $A_{1}:=\partial I_{K(v)}$ and $A_{2}:=A(v, \cdot)$ is automatically satisfied, since $D\left(A_{1}\right)=K(v)$ is bounded in $X$. Hence there exists an element $u$ which satisfies (3.2) (therefore (3.1)) for each $v \in K_{0}$. Moreover, the solution $u$ is unique by the strict monotonicity of $\tilde{A}(v, \cdot)$ and $u \in K_{0}$. Using this fact, we define a mapping $\mathrm{S}$ from $K_{0}$ into itself which assigns to each $v \in K_{0}$ the solution $u \in K_{0}$ of (3.1), i.e. $u=S v$.

Next, we show that $S$ is weakly continuous in $K_{0}$. Let $\left\{v_{n}\right\}$ be any sequence in $K_{0}$ such that $v_{n} \rightarrow v$ weakly in $X$, and put $u_{n}=S v_{n}\left(\in K_{0}\right)$ for $n=1,2, \cdots$. Now, let $\left\{u_{n_{k}}\right\}$ be any weakly convergent subsequence of $\left\{u_{n}\right\}$ and denote by $u$ the weak limit; note by condition (K2) that $u \in K(v)$. We are going to check that $u$ is a unique solution of (3.1). To do so, first observe that there is $u_{n}^{*} \in \tilde{A}\left(v_{n}, u_{n}\right)$ such that

$$
<u_{n}^{*}-g^{*}, u_{n}-w>\leq 0, \quad \forall w \in K\left(v_{n}\right)
$$

Using condition (K1), we find a sequence $\left\{\tilde{u}_{k}\right\}$ such that $\tilde{u}_{k} \in K\left(v_{n_{k}}\right)$ and $\tilde{u}_{k} \rightarrow u$ in $X$ (as $k \rightarrow \infty$ ). By the boundedness of $\tilde{A}(\cdot, \cdot)$, we may assume that $u_{n_{k}}^{*} \rightarrow u^{*}$ weakly in $X^{*}$ for some $u^{*} \in X^{*}$. Now, taking $n=n_{k}$ and $w=\tilde{u}_{k}$ in (3.3), we see that

$$
\begin{aligned}
\limsup _{k \rightarrow \infty}<u_{n_{k}}^{*}, u_{n_{k}}> & =\limsup _{k \rightarrow \infty}\left\{<u_{n_{k}}^{*}, u_{n_{k}}-\tilde{u}_{k}>+<u_{n_{k}}^{*}, \tilde{u}_{k}>\right\} \\
& \leq \limsup _{k \rightarrow \infty}\left\{<g^{*}, u_{n_{k}}-\tilde{u}_{k}>+<u_{n_{k}}^{*}, \tilde{u}_{k}>\right\} \\
& =<u^{*}, u>
\end{aligned}
$$

Therefore it follows from Proposition 2.1 that

$$
u^{*} \in \tilde{A}(v, u), \quad \lim _{k \rightarrow \infty}<u_{n_{k}}^{*}, u_{n_{k}}>=<u^{*}, u>.
$$

We go back to (3.3) with $n=n_{k}$. For any $w \in K(v)$, we use (K1) to choose a sequence $w_{k} \in K\left(v_{n_{k}}\right)$ such that $w_{k} \rightarrow w$ in $X$. Taking $n=n_{k}$ and $w=w_{k}$ in (3.3) and passing to the limit as $k \rightarrow \infty$ in (3.3), by (3.4) we obtain the variational inequality (3.1). Thus $u=S v$, and $S$ is weakly continuous in $K_{0}$.

Since $K_{0}$ is a weakly compact and convex set in $X$, we infer from the well-known fixed-point theorem for compact mappings that $S$ has at least one fixed point in $K_{0}$. This fixed point $u$ is clearly a solution of our quasi-variational ineqality $\mathrm{P}\left(g^{*}\right)$. $\diamond$

\section{(In the case of $(B))$}


We approximate $\tilde{A}(v, u)$ by $\tilde{A}_{\varepsilon}(v, u):=\tilde{A}(v, u)+\varepsilon J(u)$ for any $u, v \in X$ and with parameter $\varepsilon \in(0,1]$; note that the duality mapping $J$ from $X$ into $X^{*}$ is strictly monotone and hence $\tilde{A}_{\varepsilon}$ is a semimonotne operator such that $\tilde{A}_{\varepsilon}(v, \cdot)$ is strictly monotone for every $v \in X$. By the result of the case (A), for each $g^{*} \in X^{*}$ there exists a solution $u_{\varepsilon} \in K_{0}$ of the quasi-variational inequality

$$
u_{\varepsilon} \in K\left(u_{\varepsilon}\right), u_{\varepsilon}^{*} \in A u_{\varepsilon}, \quad<u_{\varepsilon}^{*}+\varepsilon J u_{\varepsilon}-g^{*}, u_{\varepsilon}-w>\leq 0, \quad \forall w \in K\left(u_{\varepsilon}\right),
$$

where $A$ is the operator generated by $\tilde{A}$. Now, choose a sequence $\left\{\varepsilon_{n}\right\}$, with $\varepsilon_{n} \downarrow 0$, such that $u_{n}:=u_{\varepsilon_{n}} \rightarrow u$ weakly in $X$ for some $u \in K_{0}$. Then, by conditions (K1) and (K2), we see that $u \in K(u)$ and there is a sequence $\left\{\tilde{u}_{n}\right\}$ such that $\tilde{u}_{n} \in K\left(u_{n}\right)$ and $\tilde{u}_{n} \rightarrow u$ in $X$. Moreover, by the boundedness of $\left\{u_{n}^{*}:=u_{\varepsilon_{n}}^{*}\right\}$ in $X^{*}$, we may assume that $u_{n}^{*} \rightarrow u^{*}$ weakly in $X^{*}$ for some $u^{*} \in X^{*}$. Substitute $u_{n}$ and $\tilde{u}_{n}$ for $u_{\varepsilon}$ and $w$ in (3.5) with $\varepsilon=\varepsilon_{n}$, respectively, and pass to the limit as $n \rightarrow \infty$ to get

$$
\begin{aligned}
& \limsup _{n \rightarrow \infty}<u_{n}^{*}, u_{n}-u> \\
= & \limsup _{n \rightarrow \infty}\left\{<u_{n}^{*}+\varepsilon_{n} J u_{n}, u_{n}-\tilde{u}_{n}>+<u_{n}^{*}+\varepsilon_{n} J u_{n}, \tilde{u}_{n}-u>\right\} \\
= & \limsup _{n \rightarrow \infty}\left\{<g^{*}, u_{n}-\tilde{u}_{n}>+<u_{n}^{*}, \tilde{u}_{n}-u>\right\} \\
\leq & 0 .
\end{aligned}
$$

Since $A$ is pseudo-monotone from $X$ into $X^{*}$ (cf. Proposition 2.1), it follows from the above inequality that

$$
u^{*} \in A u, \quad \lim _{n \rightarrow \infty}<u_{n}^{*}, u_{n}>=<u^{*}, u>.
$$

Now, for each $w \in K(u)$, by $(\mathrm{K} 1)$ we choose $\left\{\tilde{w}_{n}\right\}$ such that $\tilde{w}_{n} \in K\left(u_{n}\right)$ and $\tilde{w}_{n} \rightarrow w$ in $X$, and then substitute them for $w$ in (3.5) with $\varepsilon=\varepsilon_{n}$ to have

$$
<u_{n}^{*}+\varepsilon_{n} J u_{n}-g^{*}, u_{n}-\tilde{w}_{n}>\leq 0 .
$$

By (3.6), letting $n \rightarrow+\infty$ in (3.7) yields that $<u^{*}-g^{*}, u-w>\leq 0$. Thus $u$ is a solution of our quasi-variational ineqality $\mathrm{P}\left(g^{*}\right) . \diamond$

Next we proceed to the proof of Theorem 2.2.

Proof of Theorem 2.2: Put $d_{1}:=\sup _{w \in G_{0}}|w|_{X}$ and

$$
d_{2}:=\sup \left\{|w|_{X} ; w \in X, \inf _{w^{*} \in A w} \frac{<w^{*}, w-v>}{|w|_{X}} \leq\left|g^{*}\right|_{X^{*}}\left(1+d_{1}\right), \quad{ }^{\prime} v \in G_{0}\right\} .
$$

By condition (2.3), $d_{2}$ is finite. Also we put $M_{0}:=d_{1}+d_{2}+1$, and for any number $M \geq M_{0}$ consider the closed ball $B_{M}:=\left\{w \in X ;|w|_{X} \leq M\right\}$ as well as bounded closed and convex sets $K_{M}(v):=K(v) \cap B_{M}$ for all $v \in X$. Since $G_{0} \subset B_{M}$, (2.2) implies that $K_{M}(v)$ is non-empty for every $v \in X$.

We now show that conditions (K1) and (K2) in Theorem 2.1 with $K_{0}=B_{M}$ and $K(\cdot)=K_{M}(\cdot)$ are satisfied. The verification of (K2) is easy. We check condition (K1) 
for $K_{0}=B_{M}$ and $K(\cdot)=K_{M}(\cdot)$ as follows. Let $w$ be any element in $K_{M}(v)$. Then, by condition (K'1) for $K(\cdot)$, for a sequence $\left\{v_{n}\right\} \subset B_{M}$ weakly converging to $v$ there is a sequence $\left\{w_{n}\right\}$ such that $w_{n} \in K\left(v_{n}\right)$ and $w_{n} \rightarrow w$ in $X$. In the case of $|w|_{X}<M$, we see that $\left|w_{n}\right|_{X}<M$ and hence $w_{n} \in K_{M}\left(v_{n}\right)$ for all large $n$. In the case of $|w|_{X}=M$, choose an element $v_{0} \in K(v) \cap G_{0}$ and put

$$
w^{m}:=\left(1-\frac{1}{m}\right) w+\frac{1}{m} v_{0}, \quad m=1,2, \cdots .
$$

Clealy $w^{m} \in K_{M}(v)$ and $\left|w^{m}\right|_{X}<M$. Therefore, according to the above argument, for each $m$ there is a sequence $\left\{w_{n}^{m}\right\}_{n=1}^{\infty}$ such that $w_{n}^{m} \in K_{M}\left(v_{n}\right)$ and $w_{n}^{m} \rightarrow w^{m}$ in $X$ as $n \rightarrow \infty$. For each $m$ choose a number $n(m)$ so that $\left|w^{m}-w_{n}^{m}\right|_{X} \leq \frac{1}{m}$ for all $n \geq n(m)$. We may choose $\{n(m)\}_{m=1}^{\infty}$ so that $n(m-1)<n(m)$ for all $m=0,1, \cdots$, where $n(0)=1$. We put

$$
w_{n}=w_{n}^{m} \quad \text { if } \quad n(m) \leq n<n(m+1), \quad m=0,1, \cdots .
$$

It is easy to see that $w_{n} \in K_{M}\left(v_{n}\right)$ and $w_{n} \rightarrow w$ in $X$.

By the above observation we can apply Theorem 2.1 to find an element $u_{M}$ such that

$$
u_{M} \in K_{M}\left(u_{M}\right), u_{M}^{*} \in A u_{M}, \quad<u_{M}^{*}-g^{*}, u_{M}-w>\leq 0, \quad \forall w \in K_{M}\left(u_{M}\right) .
$$

Also, by condition (2.3), $\left\{u_{M} ; M \geq M_{0}\right\}$ is bounded in $X$, so that there are a sequence $\left\{M_{n}\right\}$ with $M_{n} \uparrow \infty$ and elements $u \in X, u^{*} \in X^{*}$ such that $u_{n}:=u_{M_{n}} \rightarrow u$ weakly in $X$ and $u_{n}^{*}:=u_{M_{n}}^{*} \rightarrow u^{*}$ weakly in $X^{*}$ as $n \rightarrow \infty$. We note $u \in K(u)$ by (K2). It follows from (K'1) that for each $w \in K(u)$ there is a sequence $\left\{\tilde{w}_{n}\right\}$ such that $\tilde{w}_{n} \in K\left(u_{n}\right)$ and $\tilde{w}_{n} \rightarrow w$ in $X$. In particular, denote by $\left\{\tilde{u}_{n}\right\}$ the sequence $\left\{\tilde{w}_{n}\right\}$ corresponding to $w=u$. Here, we substitute $M_{n}$ and $\tilde{u}_{n}$ for $M$ and $w$ in (3.10) to obtain $<u_{n}^{*}-g^{*}, u_{n}-\tilde{u}_{n}>\leq 0$. Hence it follows that

$$
\begin{aligned}
& \limsup _{n \rightarrow \infty}<u_{n}^{*}, u_{n}-u> \\
= & \limsup _{n \rightarrow \infty}\left\{<u_{n}^{*}-g^{*}, u_{n}-\tilde{u}_{n}>+<u_{n}^{*}, \tilde{u}_{n}-u>+<g^{*}, u_{n}-\tilde{u}_{n}>\right\} \\
\leq & 0 .
\end{aligned}
$$

By the pseudo-monotonicity of $A$ this implies that

$$
u^{*} \in A u, \lim _{n \rightarrow+\infty}<u_{n}^{*}, u_{n}>=<u^{*}, u>.
$$

By making use of these properties with (K1) and passing to the limit as $n \rightarrow \infty$ in (3.10) with $M=M_{n}$ and $w=\tilde{w}_{n}$ as above, we see that $u \in K(u)$ and $<u^{*}-g^{*}, u-w>\leq 0$ for all $w \in K(u)$. Thus $u$ is a solution of our problem $\mathrm{P}\left(g^{*}\right) . \diamond$

\section{Application to obstacle problems}

In this section, let $\Omega$ be a bounded domain in $\mathbf{R}^{N}, 1 \leq N<\infty$, with smooth boundary $\Gamma:=\partial \Omega$, and put $X:=W_{0}^{1, p}(\Omega)$ or $W^{1, p}(\Omega), 1<p<\infty$. Let $a_{i}(x, \eta, \xi), i=1,2, \cdots, N$, be functions on $\Omega \times \mathbf{R} \times \mathbf{R}^{N}$ such that 
(a1) for all $(\eta, \xi) \in \mathbf{R} \times \mathbf{R}^{N}$ the function $x \rightarrow a_{i}(x, \eta, \xi)$ is measurable on $\Omega$ for each $i=1,2, \cdot, N$;

(a2) for a.e. $x \in \Omega$ the function $(\eta, \xi) \rightarrow a_{i}(x, \eta, \xi)$ is continuous on $\mathbf{R} \times \mathbf{R}^{N}$ for each $i=1,2, \cdots, N$;

(a3) there are positive constants $c_{0}, c_{0}^{\prime}$ and $c_{1}, c_{1}^{\prime}$ such that

$$
\begin{gathered}
c_{0}|\xi|^{p-1}-c_{0}^{\prime} \leq a_{i}(x, \eta, \xi) \leq c_{1}|\xi|^{p-1}+c_{1}^{\prime}, i=1,2, \cdots, N, \\
\text { a.e. } x \in \Omega, \forall \eta \in \mathbf{R}, \forall \xi=\left(\xi_{1}, \xi_{2}, \cdots, \xi_{N}\right) \in \mathbf{R}^{N}
\end{gathered}
$$

(a4) the following monotonicity property is satisfied:

$$
\begin{gathered}
\sum_{i=1}^{N}\left(a_{i}(x, \eta, \xi)-a_{i}(x, \eta, \bar{\xi})\right)\left(\xi_{i}-\bar{\xi}_{i}\right) \geq 0 \\
\text { a.e. } x \in \Omega, \forall \eta \in \mathbf{R}, \forall \xi=\left(\xi_{1}, \xi_{2}, \cdots, \xi_{N}\right), \forall \bar{\xi}=\left(\bar{\xi}_{1}, \bar{\xi}_{2}, \cdots, \bar{\xi}_{N}\right) \in \mathbf{R}^{N} .
\end{gathered}
$$

We define a mapping $\tilde{A}_{0}(\cdot, \cdot): X \times X \rightarrow X^{*}$ by putting

$$
<\tilde{A}_{0}(v, u), w>=\sum_{i=1}^{N} \int_{\Omega} a_{i}(x, v, \nabla u) \frac{\partial w}{\partial x_{i}} d x, \quad \forall u, v, w \in X,
$$

and $A_{0} u$ by $\tilde{A}_{0}(u, u)$ for every $u \in X$. Also, let $\beta$ be a maximal monotone operator from $D(\beta)=\mathbf{R}$ into $\mathbf{R}$ such that the primitive $\hat{\beta}(r):=\int_{0}^{r} \beta(s) d s$ of $\beta$ satisfies that

$$
c_{2}|r|^{p}-c_{2}^{\prime} \leq \hat{\beta}(r) \leq c_{3}|r|^{p}+c_{3}^{\prime}, \quad \forall r \in \mathbf{R},
$$

where $c_{2}, c_{2}^{\prime}, c_{3}$ and $c_{3}^{\prime}$ are positive constants. Now, we consider an operator $\tilde{A}: X \times X \rightarrow$ $X^{*}$ given by $\tilde{A}(v, u):=\tilde{A}_{0}(v, u)+\beta(u)$ for all $v, u \in X$. It is easy to see from (4.3) and (4.4) that $\tilde{A}$ is a bounded and semimonotone operator from $X \times X$ into $X^{*}$.

Application 4.1. (Gradient obstacle problem)

Let $X=W_{0}^{1, p}(\Omega)$, and $k_{c}$ be a Lipschitz continuous real function on $\mathbf{R}$ such that

$$
0<k_{c}(r) \leq k^{*}, \quad \forall r \in \mathbf{R},
$$

where $k^{*}$ is a positive constant, and put

$$
K(v):=\left\{w \in X ;|\nabla w| \leq k_{c}(v) \text { a.e. on } \Omega\right\}, \quad \forall v \in X .
$$

Also, we set

$$
K_{0}:=\left\{w \in X ;|\nabla w| \leq k^{*} \text { a.e. on } \Omega\right\}, ;
$$

note from the Sobolev imbedding theorem that $K_{0}$ is compact in $C(\bar{\Omega})$.

Lemma 4.1. The family $\{K(v) ; v \in X\}$ and the set $K_{0}$, which are respectively given by (4.6) and (4.7), satisfy conditions (K1) and (K2). 
Proof. We prove (K2). Suppose that $v_{n} \in K_{0}, w_{n} \in K\left(v_{n}\right), v_{n} \rightarrow v$ weakly in $X$ and $w_{n} \rightarrow w$ weakly in $X$. Then, $v_{n} \rightarrow v$ in $C(\bar{\Omega})$ and hence $k_{c}\left(v_{n}\right) \rightarrow k_{c}(v)$ in $C(\bar{\Omega})$. Therefore, given $\varepsilon>0$, there exists a positive integer $n_{\varepsilon}$ such that

$$
\left|k_{c}\left(v_{n}\right)-k_{c}(v)\right| \leq \varepsilon \text { on } \Omega, \quad \forall n \geq n_{\varepsilon} .
$$

This shows that

$$
\left|\nabla w_{n}\right| \leq k_{c}\left(v_{n}\right) \leq k_{c}(v)+\varepsilon \text { a.e. on } \Omega, \quad \forall n \geq n_{\varepsilon} .
$$

Clearly the set $K_{\varepsilon}(v):=\left\{w \in X ;|\nabla w| \leq k_{c}(v)+\varepsilon\right.$ a.e. on $\left.\Omega\right\}$ is bounded, closed and convex in $X$, so that $K_{\varepsilon}(v)$ is weakly compact in $X$. Now, we derive by letting $n \rightarrow+\infty$ in (4.9) that $w \in K_{\varepsilon}(v)$. Since $\varepsilon>0$ is arbitrary, we have $w \in K(v)$. Thus (K2) holds.

Next we show (K1). Suppose that $v \in K_{0}, w \in K(v)$ and $\left\{v_{n}\right\} \subset K_{0}$ such that $v_{n} \rightarrow v$ weakly in $X$. By the compactness of $K_{0}$ in $C(\bar{\Omega})$ we have that $v_{n} \rightarrow v$ in $C(\bar{\Omega})$. Since $c w \in K(v)$ for all constant $c \in(0,1)$ and $c w \rightarrow w$ as $c \uparrow 1$ in $X$, it is enough to show the existence of a sequence $\left\{\tilde{w}_{n}\right\}$ such that $\tilde{w}_{n} \in K\left(v_{n}\right)$ and $\tilde{w}_{n} \rightarrow \tilde{w}$ in $X$, when $\tilde{w}=c w$ for any $c \in(0,1)$. In such a case, by conditions (4.5) and (4.8), we can take a small $\varepsilon>0$ so that $|\nabla \tilde{w}| \leq k_{c}(v)-\varepsilon$ a.e. on $\bar{\Omega}$. Furthermore, for this $\varepsilon>0$ we can find a positive integer $n_{\varepsilon}$ such that $k_{c}(v) \leq k_{c}\left(v_{n}\right)+\varepsilon$ for all $n \geq n_{\varepsilon}$. This implies that $|\nabla \tilde{w}| \leq k_{c}\left(v_{n}\right)$ a.e. on $\Omega$, namely $\tilde{w} \in K\left(v_{n}\right)$ for all $n \geq n_{\varepsilon}$. Now we define $\left\{\tilde{w}_{n}\right\}$ by putting

$$
\tilde{w}_{n}= \begin{cases}\tilde{w} & \text { for } n \geq n_{\varepsilon}, \\ \text { some function in } K\left(v_{n}\right) & \text { for } 1 \leq n<n_{\varepsilon} .\end{cases}
$$

Clearly this is a required sequence in condition $(\mathrm{K} 1) . \diamond$

According to Lemma 4.1, we can apply Theorem 2.1 to solve the following quasivariational inequality:

$$
\left\{\begin{array}{c}
u \in X,|\nabla u| \leq k_{c}(u) \text { a.e. on } \Omega, \quad u^{*} \in L^{q}(\Omega) \text { with } u^{*} \in \beta(u) \text { a.e. on } \Omega ; \\
\sum_{i=1}^{N} \int_{\Omega} a_{i}(x, u, \nabla u)\left(u_{x_{i}}-w_{x_{i}}\right) d x+\int_{\Omega} u^{*}(u-w) d x \leq \int_{\Omega} f(u-w) d x, \\
\forall w \in X \text { with }|\nabla w| \leq k_{c}(u) \text { a.e. on } \Omega,
\end{array}\right.
$$

where $f$ is given in $L^{q}(\Omega), \frac{1}{p}+\frac{1}{q}=1$.

Application 4.2. (Interior obstacle problem)

Let $\Omega$ be a one-dimensional bounded open interval, say $(0,1), X:=W^{1, p}(0,1), 1<$ $p<\infty$, and let $a_{1}(x, \eta, \xi)$ be a function which satisfy (a1)-(a4) with $N=1$. Let $\beta$ be the same as above.

Also, let $k_{c}(\cdot)$ be a Lipschitz continuous real function on $\mathbf{R}$ such that

$$
k_{c}(r) \leq k^{*}, \quad \forall r \in \mathbf{R},
$$

where $k^{*}$ is a constant, and put

$$
K(v):=\left\{w \in X ; w \geq k_{c}(v) \text { on }(0,1)\right\}, \quad \forall v \in X .
$$


Clearly, the constant function $k^{*}$ belongs to $K(v)$ for all $v \in X$. Futhermore, it follows from (4.1) and (4.4) that

$$
\begin{gathered}
\int_{0}^{1}\left\{a_{1}\left(x, v, v_{x}\right) v_{x}+v^{*}\left(v-k^{*}\right)\right\} d x \geq c_{4}|v|_{X}^{p}-c_{4}^{\prime}, \\
\forall v \in X, \quad \forall v^{*} \in L^{q}(0,1) \text { with } v^{*} \in \beta(v) \text { a.e. on }(0,1),
\end{gathered}
$$

where $c_{4}$ and $c_{4}^{\prime}$ are positive constants.

Lemma 4.2. The family $\{K(v) ; v \in X\}$ and the set $G_{0}:=\left\{k^{*}\right\}$ satisfy conditions (2.2), (2.3), (K'1) and (K2).

Proof. We observe that $X$ is compactly embedded in $C([0,1])$. On account of this fact, the verification of (K'1) and (K2) can be done in a way similar to that in the proof of Lemma 4.1. Also, (2.2) and (2.3) are immediately seen from (4.11), (4.12) and (4.13). $\diamond$

Now, given $f \in L^{q}(0,1)$, by applying Theorem 2.2 we find functions $u$ and $u^{*}$ such that

$$
\left\{\begin{array}{c}
u \in X, u \geq k_{c}(u) \text { on }(0,1), u^{*} \in L^{q}(0,1) \text { with } u^{*} \in \beta(u) \text { a.e. on }(0,1), \\
\int_{0}^{1}\left\{a_{1}\left(x, u, u_{x}\right)\left(u_{x}-w_{x}\right)+u^{*}(u-w)\right\} d x \leq \int_{0}^{1} f(u-w) d x \\
\forall w \in X \text { with } w \geq k_{c}(u) \text { on }(0,1) .
\end{array}\right.
$$

Application 4.3.(Boundary obstacle problem)

We consider a quasi-variational inequality with constraints on the boundary. Let $X:=W^{1, p}(0,1), 1<p<\infty$, and let $a_{1}(x, \eta, \xi)$ be a function satisfying condition (a1)(a4) with $N=1$. Let $\beta$ be the same as above. Also, let $k_{c}^{i}(\cdot), i=0,1$, be two Lipschitz continuous real functions on $\mathbf{R}$ such that

$$
k_{c}^{i}(r) \leq k^{*}, \quad \forall \in \mathbf{R}, i=0,1
$$

where $k^{*}$ is a constant. We define

$$
K(v):=\left\{w \in X ; w(i) \geq k_{c}^{i}(v(i)), i=0,1\right\}, \quad \forall v \in X
$$

and $G_{0}=\left\{k^{*}\right\}$, being the singleton set of constant function $k^{*}$. Then it is easy to see that $G_{0}$ and the family $\{K(v)\}$ satisfy conditions (2.2), (2.3), (K'1) and (K2) in the statement of Theorem 2.2. Therefore, by Theorem 2.2, for each $f \in L^{q}(0,1), \frac{1}{p}+\frac{1}{q}=1$, we find functions $u$ and $u^{*}$ such that

$$
\left\{\begin{array}{c}
u \in X, \quad u(i) \geq k_{c}(u(i)), i=0,1, \quad u^{*} \in L^{q}(0,1) \text { with } u^{*} \in \beta(u) \text { a.e. on }(0,1) \\
\int_{0}^{1}\left\{a_{1}\left(x, u, u_{x}\right)\left(u_{x}-w_{x}\right)+u^{*}(u-w)\right\} d x \leq \int_{0}^{1} f(u-w) d x \\
\forall w \in X \text { with } w(i) \geq k_{c}(u(i)), i=0,1
\end{array}\right.
$$


In Applications 4.2 and 4.3 we supposed that the space dimension of $\Omega$ is one, since we do not know whether condition (K1) or (K'1) holds or not in higher space dimensional cases.

Application 4.4. Next we consider a system of quasi-variational inequality including quasi-linear partial differential operators. Let $a_{k}\left(u_{1}, u_{2}\right), k=1,2$, be continuous functions with respect to $u_{1}$ and $u_{2}$ on $\mathbf{R} \times \mathbf{R}$ such that

$$
c_{5} \leq a_{k}\left(u_{1}, u_{2}\right) \leq c_{5}^{\prime}, \quad \forall u_{1}, u_{2} \in \mathbf{R},
$$

and let $\beta_{k}\left(u_{k}\right), k=1,2$, be maximal monotone operators from $D\left(\beta_{k}\right)=\mathbf{R}$ into $\mathbf{R}$ and the primitives $\hat{\beta}_{k}(r):=\int_{0}^{r} \beta_{k}(s) d s$ satisfy that

$$
c_{6}|r|^{p_{k}}-c_{6}^{\prime} \leq \hat{\beta}_{k}(r) \leq c_{7}|r|^{p_{k}}+c_{7}^{\prime}, \quad \forall r \in \mathbf{R}, \quad k=1,2,
$$

where $p_{k}, k=1,2$, are constants satisfying $2 \leq p_{k}<\infty$. Our problem is formulated in the space $X:=W^{1, p_{1}}(0,1) \times W^{1, p_{2}}(0,1)$, and define an operator $\tilde{A}_{0}: X \rightarrow X^{*}$ by

$$
\begin{gathered}
<\tilde{A}_{0}(v, u), w>=\int_{0}^{1} a_{1}\left(v_{1}, v_{2}\right)\left|u_{1, x}\right|^{p_{1}-2} u_{1, x} w_{1, x} d x+\int_{0}^{1} a_{2}\left(v_{1}, v_{2}\right)\left|u_{2, x}\right|^{p_{2}-2} u_{2, x} w_{2, x} d x, \\
\forall v:=\left(v_{1}, v_{2}\right), \quad u:=\left(u_{1}, u_{2}\right), \quad w:=\left(w_{1}, w_{2}\right) \in X .
\end{gathered}
$$

as well as an operator $\tilde{A}: X \rightarrow X^{*}$ by $\tilde{A}(v, u):=\hat{A}_{0}(v, u)+\left(\beta_{1}\left(u_{1}\right), \beta_{2}\left(u_{2}\right)\right)$, namely, the first and second compornent of $\tilde{A}(v, u)$ are respectively written in the form

$$
-\left(a_{1}\left(v_{1}, v_{2}\right)\left|u_{1, x}\right|^{p_{1}-2} u_{1, x}\right)_{x}+\beta_{1}\left(u_{1}\right) \text { and }-\left(a_{2}\left(v_{1}, v_{2}\right)\left|u_{2, x}\right|^{p_{2}-2} u_{2, x}\right)_{x}+\beta_{2}\left(u_{2}\right),
$$

which are multivalued, in general, because of $\beta_{1}\left(u_{1}\right)$ and $\beta_{2}\left(u_{2}\right)$. As was already seen just before Application 4.1, $\tilde{A}$ is bounded and semimonotone from $X$ into $X^{*}$. Further let $k_{k c}\left(u_{1}, u_{2}\right), k=1,2$, be Lipschitz continuous functions with respect to $u=\left(u_{1}, u_{2}\right)$ on $\mathbf{R} \times \mathbf{R}$ such that $k_{k c} \leq k^{*}$ on $\mathbf{R} \times \mathbf{R}$ for a constant $k^{*}$. Then, given $f_{k} \in L^{q_{k}}(0,1)$, $\frac{1}{p_{k}}+\frac{1}{q_{k}}=1, k=1,2$, by virtue of Theorem 2.2 there exist vector functions $u=\left(u_{1}, u_{2}\right) \in X$ and $u^{*}=\left(u_{1}^{*}, u_{2}^{*}\right) \in L^{q_{1}}(0,1) \times L^{q_{2}}(0,1)$ such that

$$
\left\{\begin{array}{c}
u_{k} \geq k_{k c}\left(u_{1}, u_{2}\right) \text { on }(0,1), \quad u_{k}^{*} \in \beta_{k}\left(u_{k}\right) \text { a.e. on }(0,1), \quad k=1,2 \\
\int_{0}^{1} a_{k}\left(u_{1}, u_{2}\right)\left|u_{k, x}\right|^{p_{k}-2}\left(u_{k, x}-w_{k, x}\right) d x+\int_{0}^{1} u_{k}^{*}\left(u_{k}-w_{k}\right) d x \leq \int_{0}^{1} f_{k}\left(u_{k}-w_{k}\right) d x \\
\forall w_{k} \in W^{1, p_{k}}(0,1) \text { with } w_{k} \geq k_{k c}\left(u_{1}, u_{2}\right) \text { on }(0,1), \quad k=1,2 .
\end{array}\right.
$$

\section{Application to problems with non-local constraints}

Let $\Omega$ be a bounded domain in $\mathbf{R}^{N}, 1 \leq N<\infty$, with smooth boundary $\Gamma:=\partial \Omega$, and let $X:=W^{1, p}(\Omega)$ or $W_{0}^{1, p}(\Omega), 1<p<\infty$. Let $a_{i}(x, \eta, \xi), i=1,2, \cdots, N$, and $\beta$ be as in the previous section; conditions (4.1), (4.2) and (4.4) are satisfied as well. Furthmore let $k_{c}(\cdot)$ be a Lipschitz continuous function on $\mathbf{R}$ with bounded Lipschitz continuous derivative $k_{c}^{\prime}(\cdot)$ on $\mathbf{R}$; condition (4.11) is satisfied as well. 
Given a singlevalued compact mapping $\Lambda: X \rightarrow X$, we define constraint sets $K(v)$ in $X$ by

$$
K(v):=\left\{w \in X ; w \geq k_{c}(\Lambda v) \text { a.e. on } \Omega\right\}, \quad \forall v \in X .
$$

Clearly, $K(v)$ is non-empty, closed and convex in $X$ for every $v \in X$.

Lemma 5.1. The family $\{K(v) ; v \in X\}$ given by (5.1) and the set $G_{0}:=\left\{k^{*}\right\}$ satisfy conditions (K'1) and (K2).

Proof. Assume that $v_{n} \rightarrow v$ weakly in $X$ and let $w$ be any function in $K(v)$, namely $w \geq k_{c}(\Lambda v)$ a.e. on $\Omega$. We note that $\Lambda v_{n} \rightarrow \Lambda v$ in $X$ and hence $k_{c}\left(\Lambda v_{n}\right) \rightarrow k_{c}(\Lambda v)$ in $X$. Putting $w_{n}=w-k_{c}(\Lambda v)+k_{c}\left(\Lambda v_{n}\right)$, we see that $w_{n} \in K\left(v_{n}\right), w_{n} \rightarrow w$ in $X$. Thus (K'1) is verified. Next, assume that $w_{n} \in K\left(v_{n}\right), w_{n} \rightarrow w$ weakly in $X$ and $v_{n} \rightarrow v$ weakly in $X$. Then $w_{n} \rightarrow w$ and $v_{n} \rightarrow v$ in $L^{p}(\Omega)$ as well as $k_{c}\left(\Lambda v_{n}\right) \rightarrow k_{c}(\Lambda v)$ in $L^{p}(\Omega)$. Hence $w \geq k_{c}(\Lambda v)$ a.e. on $\Omega$, that is $w \in K(v)$. Thus (K2) is obtained. $\diamond$

For the same mapping $\tilde{A}:=\tilde{A}_{0}+\beta$ as in the previous section, all the conditions of Theorem 2.2 are verified. Therefore, given a function $f \in L^{q}(\Omega), \frac{1}{p}+\frac{1}{q}=1$, there exists a function $u \in X$ and $u^{*} \in L^{q}(\Omega)$ such that

$$
\left\{\begin{array}{c}
u \in X, u \geq k_{c}(\Lambda u) \text { a.e. on } \Omega, u^{*} \in \beta(u) \text { a.e. on } \Omega \\
\sum_{i=1}^{N} \int_{\Omega} a_{i}(x, u, \nabla u)\left(u_{x_{i}}-v_{x_{i}}\right) d x+\int_{\Omega} u^{*}(u-w) d x \leq \int_{\Omega} f(u-w) d x \\
\forall w \in X \text { with } w \geq k_{c}(\Lambda u) \text { a.e. on } \Omega \text {. }
\end{array}\right.
$$

Next, consider typical examples of the mapping $\Lambda$.

(Example 5.1) Consider the case when $p=2$ and $X:=W^{1,2}(\Omega)$. Let $\nu$ be a positive number. Then, for each $v \in X$, the boundary value problem

$$
-\nu \Delta v+v=u \text { in } \Omega, \quad \frac{\partial v}{\partial n}=0 \text { on } \Gamma,
$$

has a unique solution $v$ in $W^{2,2}(\Omega)$. Now, we define $\Lambda: X \rightarrow X$ by $v=\Lambda u$, where $v$ is the solution of (5.3) for $u \in X$. Since $\Lambda$ is bounded and linear from $X$ into $W^{2,2}(\Omega)$, we see that $\Lambda$ is compact from $X$ into itself; in fact, $\Lambda=(I-\nu \Delta)^{-1}$. In this case, given $f \in L^{2}(\Omega)$, the quasi-variational inequality (5.2) with $p=2$ is a system to find $u \in W^{1,2}(\Omega)$ with $u^{*} \in L^{2}(\Omega)$ and $v \in W^{2,2}(\Omega)$ such that

$$
\left\{\begin{array}{l}
u \geq k_{c}(v) \text { a.e. on } \Omega, \quad u^{*} \in \beta(u) \text { a.e. on } \Omega \\
\sum_{i=1}^{N} \int_{\Omega} a_{i}(x, u, \nabla u)\left(u_{x_{i}}-w_{x_{i}}\right) d x+\int_{\Omega} u^{*}(u-w) d x \leq \int_{\Omega} f(u-w) d x \\
\forall w \in X \text { with } w \geq k_{c}(v) \text { a.e. on } \Omega \\
-\nu \Delta v+v=u \text { a.e. on } \Omega \\
\frac{\partial v}{\partial n}=0 \text { a.e. on } \Gamma
\end{array}\right.
$$


and by virtue of Theorem 2.2 the above system has at least one solution $\left\{u_{\nu}, v_{\nu}\right\}$ for each $\nu \in(0,1]$. Also, see [12] for a related non-local quasi-variational inequality.

Proposition 5.1. Assume that the space dimension is one, $p=2$ and $\Omega=(0,1)$. Let $\left\{u_{\nu}, v_{\nu}\right\}_{\nu \in(0,1]}$ be a family of solutions of (5.4). Then $\left\{u_{\nu}, v_{\nu}\right\}$ is bounded in $W^{1,2}(\Omega) \times$ $W^{1,2}(\Omega)$, and for any weak limit $u$ of $\left\{u_{\nu}\right\}$ as $\nu \downarrow 0$ is a solution of the interior obstacle problem (4.14).

Proof. By testing $k^{*}$ of (4.11) in quasi-variational inequality in (5.4) with $u=u_{\nu}$, it is easy to see that $\left\{u_{\nu}\right\}_{\nu \in(0,1]}$ is bounded in $W^{1,2}(0,1)$, hence is relatively compact in $C([0,1])$. Now, let $u$ be any weak limit of $\left\{u_{\nu}\right\}$ and choose a sequence $\nu_{n}$ with $\nu_{n} \downarrow 0$ so that $u_{n}:=u_{\nu_{n}} \rightarrow u$ weakly in $W^{1,2}(0,1)$, hence $u_{n}:=u_{\nu_{n}} \rightarrow u$ uniformly on $[0,1]$, as $n \rightarrow \infty$. Putting $v_{n}:=v_{\nu_{n}}$ and multiplying $-\nu_{n} \Delta v_{n}+v_{n}=u_{n}$ by $v_{n}$ and $-\Delta v_{n}$, we obtain

$$
\begin{gathered}
\int_{0}^{1}\left|\nabla v_{n}\right|^{2} d x+\int_{0}^{1}\left|v_{n}\right|^{2} d x \leq \int_{0}^{1}\left|v_{n}\right|\left|u_{n}\right| d x, \\
\nu_{n} \int_{0}^{1}\left|\Delta v_{n}\right|^{2} d x+\int_{0}^{1}\left|\nabla v_{n}\right|^{2} d x \leq \int_{0}^{1}\left|\nabla v_{n}\right|\left|\nabla u_{n}\right| d x,
\end{gathered}
$$

whence $\left\{\nu_{n}\left|\Delta v_{n}\right|_{L^{2}(0,1)}^{2}\right\}$ is bounded and $\left\{v_{n}\right\}$ is bounded in $W^{1,2}(0,1)$. Since $v_{n}-u_{n}=$ $\nu_{n} \Delta v_{n} \rightarrow 0$ in $L^{2}(0,1)$ and $u_{n} \geq k_{c}\left(v_{n}\right)$, it follows from the above esitimates that

$$
v_{n} \rightarrow u \text { weakly in } W^{1,2}(\Omega) \text { and uniformly on }[0,1]
$$

as well as

$$
k_{c}\left(v_{n}\right) \rightarrow k_{c}(u) \text { uniformly on }[0,1], \quad u \geq k_{c}(u) \text { on }(0,1) .
$$

Let $w$ be any function with $w \geq k_{c}(u)$ on $(0,1)$. Given $\varepsilon>0$, choose a positive integer $n_{\varepsilon}$ so that

$$
w+\varepsilon \geq k_{c}(u)+\varepsilon \geq k_{c}\left(v_{n}\right), \quad u+\varepsilon \geq k_{c}(u)+\varepsilon \geq k_{c}\left(v_{n}\right), \quad \text { on }(0,1), \quad \forall n \geq n_{\varepsilon} ;
$$

this is possible on account of (5.5) and (5.6). Now, take $u+\varepsilon$ as $w$ the quasi-variational inequality in (5.4) which $u_{n}$ satisfies, to get

$$
\int_{0}^{1} a_{1}\left(x, u_{n}, u_{n, x}\right)\left(u_{n, x}-u_{x}\right) d x+\int_{0}^{1} u_{n}^{*}\left(u_{n}-u-\varepsilon\right) d x \leq \int_{0}^{1} f\left(u_{n}-u-\varepsilon\right) d x,
$$

where $u_{n}^{*} \in L^{2}(0,1)$ is a function satisfying $u_{n}^{*} \beta\left(u_{n}\right)$ a.e. on $(0,1)$. Then we have

$$
H:=\limsup _{n \rightarrow \infty} \int_{0}^{1} a_{1}\left(x, u_{n}, u_{n, x}\right)\left(u_{n, x}-u_{x}\right) d x+\int_{0}^{1} u_{n}^{*}\left(u_{n}-u\right) d x \leq M_{0} \varepsilon,
$$

where $M_{0}$ is a positive constant. Since $\varepsilon>0$ is arbitrary, it holds that $H \leq 0$. This inequality implies by the pseudo-monotonicity property that

$$
\lim _{n \rightarrow \infty} \int_{0}^{1} a_{1}\left(x, u_{n}, u_{n, x}\right)\left(u_{n, x}-u_{x}\right) d x+\int_{0}^{1} u_{n}^{*}\left(u_{n}-u\right) d x=0
$$

and

$$
\lim _{n \rightarrow \infty} \int_{0}^{1} a_{1}\left(x, u_{n}, u_{n, x}\right) \tilde{w}_{x} d x+\int_{0}^{1} u_{n}^{*} \tilde{w} d x=\int_{0}^{1} a_{1}\left(x, u, u_{x}\right) \tilde{w}_{x} d x+\int_{0}^{1} u^{*} \tilde{w} d x
$$




$$
\forall \tilde{w} \in W^{1,2}(0,1),
$$

where $u^{*} \in L^{2}(0,1)$ with $u^{*} \in \beta(u)$ a.e. on $(0,1)$. Going back to the quasi-variational inequality (5.4) with $\nu=\nu_{n}$ and $u=u_{n}$ and $v=v_{n}$ for any $n \geq n_{\varepsilon}$, we obtain by (5.7)

$$
\int_{0}^{1} a_{i}\left(x, u_{n}, u_{n, x}\right)\left(u_{n, x}-w_{x}\right) d x+\int_{0}^{1} u_{n}^{*}\left(u_{n}-w-\varepsilon\right) d x \leq \int_{0}^{1} f\left(u_{n}-w-\varepsilon\right) d x
$$

for all $w \in W^{1,2}(0,1)$ with $w \geq k_{c}(u)$ on $(0,1)$. Letting $n \rightarrow \infty$ in this equality and using (5.8) and (5.9), we see that

$$
H(u, w):=\int_{0}^{1} a_{i}\left(x, u, u_{x}\right)\left(u_{x}-w_{x}\right) d x+\int_{0}^{1} u^{*}(u-w) d x-\int_{0}^{1} f(u-w) d x \leq M_{0} \varepsilon
$$

for all $w \in W^{1,2}(0,1)$ with $w \geq k_{c}(u)$ on $(0,1)$. By the arbitrariness of $\varepsilon>0$ we conclude that $H(u, w) \leq 0$ and $u$ is a solution of quasi-variational inequality (4.14). $\diamond$

(Example 5.2) The second example of $\Lambda$ is given as an integral operator as follows. Let $\rho(\cdot, \cdot, \cdot)$ be a smooth function on $\mathbf{R}^{N} \times \mathbf{R}^{N} \times \mathbf{R}$ such that

$$
|\rho(x, y, r)| \leq c_{4}|r|+c_{4}^{\prime}, \quad \forall x, y \in \mathbf{R}^{N}, \forall r \in \mathbf{R},
$$

where $c_{4}$ and $c_{4}^{\prime}$ are positive constants. Then we define $\Lambda: X:=W^{1, p}(\Omega) \rightarrow X$ by

$$
(\Lambda v)(x)=\int_{\Omega} \rho(x, y, v(y)) d y, \quad \forall x \in \Omega, \quad \forall v \in X .
$$

Clearly $\Lambda$ is compact from $X$ into itself.

Now we give some concrete examples of integral operator $\Lambda$.

(Case 1) We consider as $\Lambda$ the usual convolution operator by means of mollifier $\rho_{\varepsilon}$ with real parameter $0<\varepsilon<1$. Let

$$
\rho_{\varepsilon}(x, y):=\frac{1}{\varepsilon^{N}} \rho_{0}\left(\frac{x-y}{\varepsilon}\right), \forall x, y \in \mathbf{R}^{N},
$$

where

$$
\rho_{0}(x):= \begin{cases}c \cdot \exp \left\{-\frac{1}{1-|x|^{2}}\right\}, & \text { if }|x|<1 \\ 0, & \text { otherwise }\end{cases}
$$

the constant $c>0$ being chosen so that $\int_{\mathbf{R}^{N}} \rho_{0} d x=1$. Then, the mapping $\Lambda=\Lambda_{\varepsilon}$ is given by

$$
\left(\Lambda_{\varepsilon} v\right)(x)=\int_{\Omega} \rho_{\varepsilon}(x-y) v(y) d y, \quad \forall v \in X
$$

as well as the constraint set $K(v)=K_{\varepsilon}(v)$ is of the form:

$$
K_{\varepsilon}(v)=\left\{w \in X ; w \geq k_{c}\left(\int_{\Omega} \rho_{\varepsilon}(\cdot-y) v(y) d y\right) \text { a.e. on } \Omega\right\} .
$$

According to the above existence result, for each $\varepsilon>0$ problem (5.2) with $\Lambda:=\Lambda_{\varepsilon}$ has at least one solution $u_{\varepsilon}\left(\in K_{\varepsilon}\left(u_{\varepsilon}\right)\right)$. For the family $\left\{u_{\varepsilon}\right\}$ of solutions we see by taking $w=k^{*}$ 
in inequality (5.2) that $\left\{u_{\varepsilon}\right\}_{0<\varepsilon<1}$ is bounded in $X$. It is a quite interesting question what happens as $\varepsilon \rightarrow 0$.

Proposition 5.2. Assume that the space dimension is one and $\Omega=(0,1)$. Let $\left\{u_{\varepsilon}\right\}_{\varepsilon \in(0,1]}$ be a family of solutions of (5.2) with $\Lambda=\Lambda_{\varepsilon}$. Then $\left\{u_{\varepsilon}\right\}$ is bounded in $W^{1, p}(0,1)$ and any weak limit $u$ of $\left\{u_{\varepsilon}\right\}$ as $\varepsilon \downarrow 0$ is a solution of the interior obstacle problem (4.14).

Proof. We take $w \equiv k^{*}$ in (5.2) with $u=u_{\varepsilon}$ and $\Lambda=\Lambda_{\varepsilon}$ to get

$$
\int_{0}^{1} a_{1}\left(x, u_{\varepsilon}, u_{\varepsilon, x}\right) u_{\varepsilon, x} d x+\int_{0}^{1} u_{\varepsilon}^{*}\left(u_{\varepsilon}-k^{*}\right) d x \leq \int_{0}^{1} f\left(u_{\varepsilon}-k^{*}\right) d x .
$$

It is easy to see from this inequality that $\left\{u_{\varepsilon}\right\}$ is bounded in $W^{1, p}(0,1)$. Now let $u$ be any weak limit of $\left\{u_{\varepsilon}\right\}$ in $W^{1, p}(0,1)$ and choose a sequence $\varepsilon_{n} \downarrow 0$ so that $u_{n}:=u_{\varepsilon_{n}} \rightarrow u$ weakly in $W^{1, p}(0,1)$ and uniformly on $[0,1]$. In this case we have that $\Lambda_{\varepsilon_{n}} u_{n} \rightarrow u$ uniformly on $[0,1]$ and $u \geq k_{c}(u)$ on $(0,1)$. Therefore, in a way similar to that of Proposition 5.1, we can prove that $u$ is a solution of the quasi-variational inequality (4.14). $\diamond$

(Case 2) Consider $\Omega=(0,1)$ and define $\rho(x, y)$ by

$$
\rho(x, y):= \begin{cases}s(x-y), & \text { if } y \in[x, 1), \\ 0, & \text { if } y \in(0, x),\end{cases}
$$

where $s(\cdot)$ is a non-negative smooth function on $\mathbf{R}$ such that $s(x)=0$ for $x \leq 0$. Then, we define $\Lambda: X:=W^{1, p}(0,1) \rightarrow X$ by

$$
(\Lambda v)(x)=\int_{0}^{1} s(x-y) v(y) d y, \quad \forall v \in X .
$$

where $k_{c}(\cdot)$ is as before. In this case, by the definition of quasi-variational inequality any solution $u$ of (5.2) has to satisfy that

$$
u(x) \geq k_{c}\left(\int_{0}^{1} s(x-y) u(y) d y\right), \quad \text { a.e. } x \in(0,1) .
$$

As is easily understood, the behaviour of $u$ satisfying (5.12) is controlled by that of $s(\cdot)$, and some suitable classes of functions $s(\cdot)$ are expected to play a role of an effective control space, for instance, in optimal design problems. In this sense, it is interesting to derive necessary conditions of $u$ in order to satisfy (5.12). For instance, it is easily checked from (5.12) that $u(1) \geq k_{c}(0)$, because, by (5.11), $s(1-y)=0$ for all $y \in(0,1)$. This means that it may be possible to control the solution $u$ of problem (5.2) by appropriate choice of convolution kernel $s(\cdot)$.

(Case 3) In case $\rho(x, y)$ is constant $|\Omega|$ on $\mathbf{R}^{N} \times \mathbf{R}^{N}$, where $|\Omega|$ is the volume of $\Omega$, we define

$$
(\Lambda v)(x)=\frac{1}{|\Omega|} \int_{\Omega}|v(y)| d y, \quad \forall x \in \Omega, \quad \forall v \in X:=W^{1, p}(\Omega) .
$$

In this case, we see that $\Lambda$ is a continuous mapping from the weak topology of $X$ into $\mathbf{R}$, which is regarded as a compact mapping from $X$ into itself. 


\section{References}

1. C.Baiocchi and A.Capelo, Variational and Quasivariational Inequalities, John Wiley and Sons, Chichester-New York-Brisbane-Toronto-Singapore, 1984.

2. A. Bensoussan and J.L. Lions, Nouvelle formulation de problémes de controle impulsionnel et applications, C. R. Acad. Sci. Paris Sér. A, 276(1973), 1189-1192.

3. A. Bensoussan, M. Goursat, J.L. Lions, Contrôle impulsionnel et inéquations quasivariationnelles stationnaires, C. R. Acad. Sci. Paris Ser. A, vol.276(1973), 12791284.

4. H.Brézis, Équations et inéaires dans les espaces vectoriels en dualité, Ann. Inst. Fourier, Grenoble, 18 (1968), 115-175.

5. F.E. Browder, Nonlinear monotone operators and convex sets in Banach spaces, Bull. Amer. Math. Soc., 71(1965), 780-785.

6. J.L. Joly and U. Mosco, Sur les inéquations quasi-variationnelles, C. R. Acad. Sci. Paris Ser. A, 279(1974), 499-502.

7. J.L. Joly and U. Mosco, A propos de l'existence et de la régularité des solutions de certaines inéquations quasi-variationnelles, J. Functional Analysis, 34(1979), $107-$ 137.

8. N.Kenmochi, Nonlinear operators of monotone type in reflexive Banach spaces and nonlinear perturbations, Hiroshima Math. J., 4(1974), 229-263.

9. T. Laetsch, A uniqueness theorem for elliptic quasi-variational inequalities, J. Functional Analysis, 18(1975), 286-287.

10. J.L. Lions and G. Stampacchia, Variational inequalities, Comm. Pure Appl. Math., 20(1967), 493-519.

11. U. Mosco, Convergence of convex sets and of variational inequalities, Advances Math., 3(1969), 510-585.

12. N. Nassif and K. Malla, Étude de l'existence de la solution d'une inégalité quasi variationnelle apparaissant dans la théorie des semi-conducteurs, C. R. Acad. Sci. Paris Serie 1, 294(1982), 119-122.

13. L. Tartar, Inéquations quasi variationnelles abstraites, C. R. Acad. Sci. Paris Ser. A, 278(1974), 1193-1196. 